The future in the present

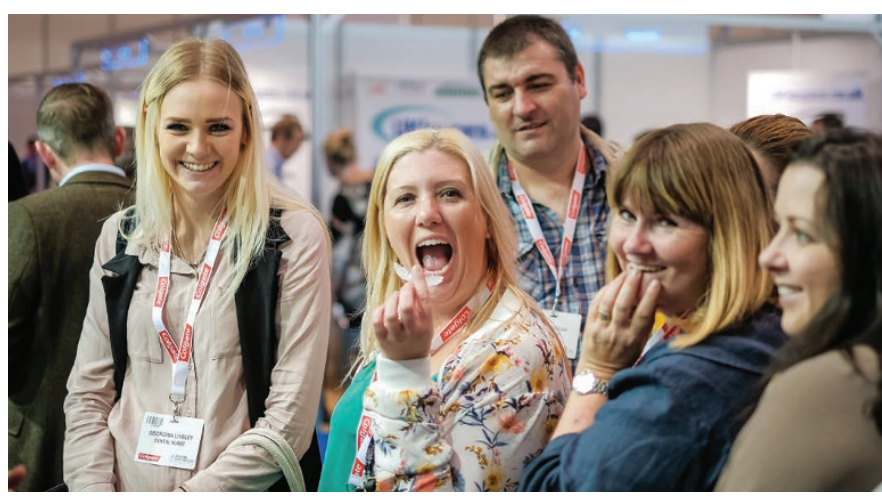

As a part of the largest dental show in the UK, Dental Showcase will this year for the first time include a 'dental surgery of the future' on the show floor, featuring a wide range of the latest solutions and products being demonstrated live. The purpose-built practice will include a reception area, patient information zone and the piece-deresistance - a fully functioning surgery with arena seating for visitors to experience the latest kit within the setting in which it will be used.

Showcase will again host thousands of professionals as they discover the latest dental innovations across an unrivalled selection of exhibitor stands. Dental team members will enjoy personalised product demonstrations, be able to snap up unbeatable deals and top up their CPD, all under one roof.

While the main thrust of the show will focus on the exhibits, the CPD presentations will include keynotes on the future of dentistry, live demonstrations on how to handle medical emergencies, innovations in $\mathrm{X}$-rays, $3 \mathrm{D}$ printing, how to make the right purchasing decisions, IRMER and much more.

Witness the future in the present - Dental Showcase 2017 is a must for everyone who needs to be in the know. To register your place at the event, which will take place at Birmingham NEC from 19-21 October 2017, log onto http://www.dentalshowcase.com/register.

\section{Make a real difference to your patients}

Dentine hypersensitivity can be extremely uncomfortable and may have a significant impact on quality of life for your patients. A new unique study using an Oral Health Impact Profile (OHIP-49) to measure dentine hypersensitivity scores demonstrated Colgate Sensitive Pro-Relief provides clinically significant improvements in Quality of Life, compared to a negative control at 4,8 and 24 weeks $(\mathrm{p}=0.005) .{ }^{1}$ This pioneering study with 273 patients is the first double-blind clinical trial to show that a toothpaste technology can make a real difference to the lives of patients suffering from dentine hypersensitivity. ${ }^{1}$

Colgate Sensitive Pro-Relief contains unique

Pro-Argin technology. Pro-Argin enhances the natural mechanisms of tubule occlusion to deposit a dentine-like material, containing calcium and phosphate, within the tubules. Resistant to acid attack, Pro-Argin forms a protective layer on the dentine surface ${ }^{2}$ providing both instant [when toothpaste is applied directly to each affected tooth for one minute $]^{3,4}$ and long lasting relief from the pain of sensitive teeth. ${ }^{4,5}$

By simply changing toothpaste to Colgate Sensitive ProRelief your patients can experience consistent long-lasting pain relief $^{4,5}$ whilst maintaining recommended cavity protection. ${ }^{6}$

Recommend Colgate Sensitive Pro-Relief with confidence and make a real difference to your patients suffering from dentine hypersensitivity.

1. Gernhardt C R et al. Effect of Pro-Argin technology on oral health-related quality of life and dentine hypersensitivity vs. negative control over 24 weeks. Poster presented at FDI 2016.

2. Petrou l et al. Am J Dent 2009; 22 (Spec Iss A): 23A-31A.

3. Docimo R et al. J Clin Dent 2009; (Spec Iss A): 17-22.

4. Hamlin D et al. Am. J Dent 2009; 22 (Spec Iss A): 16A-20A.

5. Nathoo $S$ et al. J Clin Dent 2009; 20 (Spec Iss): 123-130.

6. Recommended fluoride level (1350-1500 ppm) for caries prevention in Delivering better oral health. An evidence-based toolkit for prevention. 3rd ed. Public Health England, June 2014.

\section{Bespoke, 3D-printed medical implants}

Ortho Baltic, a Lithuanian medical company based in Kaunas, is the first manufacturer of patient-specific, temporomandibular joint endoprostheses in the Baltic states and one of only three in Europe. The implants replace loss or lack of functionality of jaw movement. To underpin their production, in 2012 the firm invested in additive manufacturing (AM) technology and recently acquired CT inspection equipment from Nikon Metrology for quality control.

Ortho Baltic aims to move away from a 'one implant fits all' mindset towards tailored solutions and to make patient-specific implants affordable and readily available for all patients and national healthcare systems. It also designs and produces patient specific

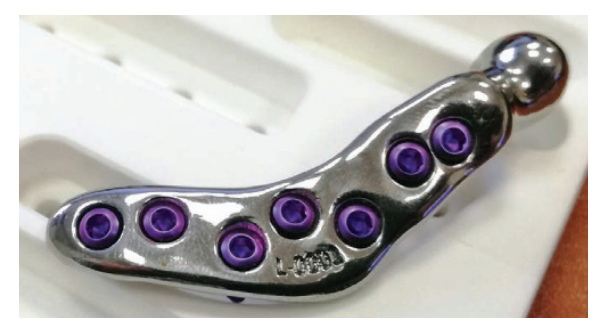

joints, cranial and spinal implants, bone plating systems, dental and jaw restoration implants and surgical guides.

The Implant Design and Development Centre uses various AM processes for the different types of components produced. Typically, implants are made from medical Grade- 5 titanium alloy $\mathrm{Ti}_{6} \mathrm{Al}_{4} \mathrm{~V}$ by direct laser metal sintering. For its surgical guides and implant models, a biocompatible polymer PA 2200 (also known as Nylon-12) is selective laser sintered.
$\mathrm{X}$-ray plays an important role in the process from start to end. Medical CT scans are the basis for preparing the CAD model, while inspection of AM parts is carried out with a Nikon Metrology industrial, high voltage, micro-CT scanner.

The Nikon Metrology XT H 225 micro-CT scanner for quality inspection at Kaunas has a $225 \mathrm{kV}$ micro-focus source that reveals non-destructively any voids, cracks and other defects of the complex internal features of the AM components.

The CT system is helping Ortho Baltic to make tailored implants affordable and easily available. In comparison to standard implants previously sourced from the USA, the firm has been able to produce its implants to the same high quality at almost half the cost.

www.nikonmetrology.com 